\title{
Spontaneous benign pneumoperitoneum complicating scleroderma in the absence of pneumatosis cystoides intestinalis
}

\author{
N.J.M. London, R.G. Bailey and A.W. Hall \\ Department of Surgery, Glenfield General Hospital, Leicester, UK.
}

\begin{abstract}
Summary: We describe a 64 year old woman with a 3-year history of scleroderma who presented as an emergency with increasing painless abdominal distention. Radiological investigations revealed a pneumoperitoneum in the absence of either visceral perforation or pneumatosis cystoides intestinalis. This is only the fourth report of spontaneous benign pneumoperitoneum complicating scleroderma without pneumatosis cystoides intestinalis. The possible aetiology of this condition is discussed.
\end{abstract}

\section{Introduction}

Serious gastrointestinal involvement is present in approximately $50 \%$ of patients with scleroderma. ${ }^{1}$ Spontaneous pneumoperitoneum is a rare complication of the disease and is usually associated with pneumatosis cystoides intestinalis. ${ }^{2}$ We report a case of spontaneous pneumoperitoneum in a patient with scleroderma in whom there was no evidence of either visceral perforation nor of pneumatosis cystoides intestinalis.

\section{Case report}

A 64 year old woman presented as an emergency with constipation, vomiting and increasing painless abdominal distension. Three years previously she had been diagnosed as suffering from scleroderma and had been admitted to hospital two years later with a self-limiting episode of intestinal obstruction. Contrast studies of the oesophagus, small and large bowel at that time had shown a dilated atonic oesophagus with free gastro-oesophageal reflux and small bowel dilatation with an increased transit time. There was no evidence of pneumatosis cystoides intestinalis. Physical examination on this occasion revealed a typical sclerodermatous facies with sclerodactyly and dry gangrene of the distal left ring finger. She was apyrexial with a pulse rate of 88 per minute and blood pressure of $100 /$

Correspondence: N.J.M London, M.R.C.P., F.R.C.S., Department of Surgery, Clinical Sciences Building, Leicester Royal Infirmary, PO Box 65, Leicester LE2 7LX, UK.

Accepted: 16 May 1989
$60 \mathrm{mmHg}$. The abdomen was grossly distended, soft, non-tender and tympanitic on percussion. Urgent laboratory investigations showed a normal haemoglobin and white cell count. Abdominal (Figure 1) and chest X-rays revealed a large pneumoperitoneum and although there was small bowel and colonic dilatation there was no radiological evidence of a localized point of obstruction within the bowel.

Because the clinical picture was not compatible with a visceral perforation a peritoneal lavage was performed. This yielded odourless gas with the return of clear lavage fluid and a reduction in the patient's abdominal distension. It was concluded that the diagnosis was that of benign spontaneous peritoneum and conservative treatment was initiated. Her condition improved over the next week such that she was able to eat normally. Repeat contrast studies including a gastrograffin meal, small bowel enema and large bowel enema did not show any evidence of visceral perforation nor of pneumatosis cystoides intestinalis.

The patient's condition gradually improved until 19 days after admission when she suddenly developed a dense right-sided hemiplegia and died. Permission for a post-mortem examination was sought but was not obtained.

\section{Discussion}

Gastrointestinal involvement in scleroderma is characterized by atrophy of the muscularis propria and its replacement by collagen tissue. These changes lead to decreased peristaltic activity with 


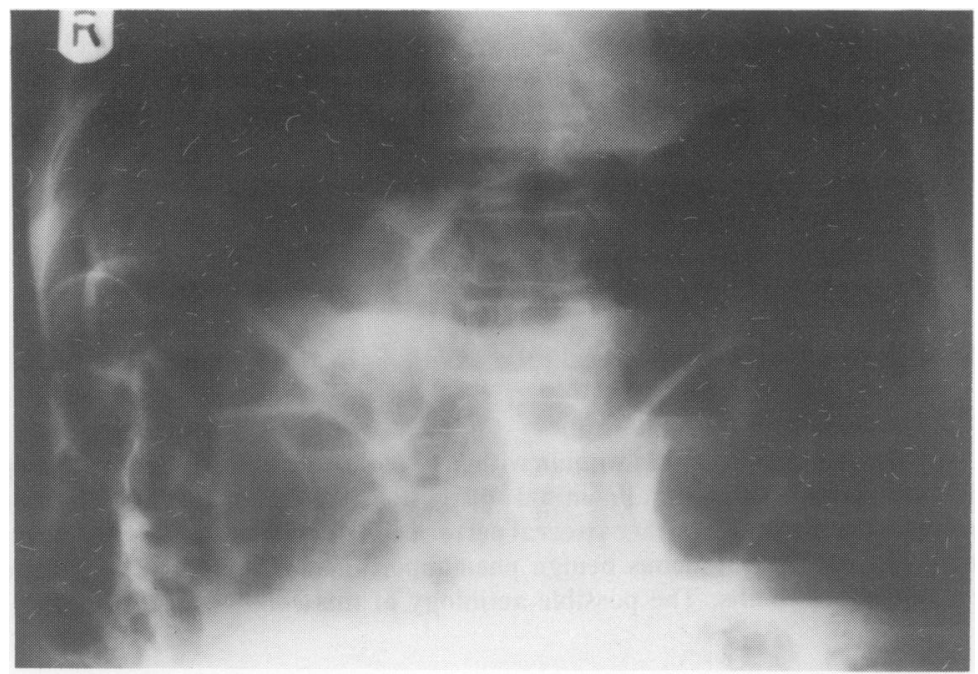

Figure 1 Abdominal X-ray showing pneumoperitoneum with small and large bowel dilatation.

consequent stasis and distension. Although any part of the gastrointestinal tract may be involved, the organs involved in decreasing order of frequency are the oesophagus, small bowel, colon and stomach. ${ }^{3}$ The oesophageal motor abnormalities may lead to gastro-oesophageal reflux, oesophagitis and stricture. Oesophageal moniliasis is not infrequent in scleroderma. Abnormalities of small intestinal motility are present in $40 \%$ of patients and may lead to atony, dilatation and large, wide-necked diverticula. These diverticula represent localized areas of dilatation due to smooth muscle atrophy. Colonic involvement, demonstrated by barium enema, occurs in 10 to $50 \%$ of patients, ${ }^{3}$ the commonest finding being sacculations. A variety of complications may supervene in patients with scleroderma. Intestinal ischaemia and perforation may be due to vasculitis. The colonic motor disturbance predisposes to the formation of faecaliths, which, if they become lodged in a diverticulum, produce an appearance that can be mistaken for a colonic tumour. Intestinal malabsorption may result from bacterial overg-

\section{References}

1. Poirier, T.J. \& Rankin, G.B. Gastrointestinal manifestations of progressive systemic scleroderma based on a review of 364 cases. Am J Gastroenterol 1972, 58: 30-44.

2. Fallon, R.H. Pneumatosis cystoides intestinalis, associated with scleroderma and presenting with pneumoperitoneum. Mo Med 1967, 64: 117-118.

3. Cohen, G., Laufer, I., Snape, W.J., Yih-fu. S., Levine, G.M. \& Jimenez. S. The gastrointestinal manifestations of scleroderma: pathogenesis and management. Gastroenterology 1980, 79: 155-166. rowth, increased thickness of the unstirred water layer, or thickening, fragmentation and collagenous infiltration of the muscularis mucosa.

Spontaneous pneumoperitoneum is a rare complication in patients with intestinal scleroderma and was first reported by Fallon in a patient with scleroderma and pneumatosis cystoides intestinalis. ${ }^{2}$ There have only been three previous reports of spontaneous pneumoperitoneum in the absence of pneumatosis cystoides intestinalis. ${ }^{4-6}$ The aetiology of the pneumoperitoneum in these circumstances is obscure although it has been suggested that recurrent microperforations along the gastrointestinal tract allow air to leak into the peritoneal cavity. ${ }^{6}$

It is important that doctors should be aware of spontaneous benign pneumoperitoneum in patients with scleroderma and that the absence of pneumatosis cystoides intestinalis does not exclude the diagnosis. Unnecessary surgery in these patients will not only compromise their intestinal function but also place their lives at risk.

4. Bloch, F., Leport, J., Mallet, L., Fiessinger, J.W., Housset, E. \& Petite, J.P. Spontaneous pneumoperitoneum in systemic sclerosis. Gastroenterol Clin Biol 1984, 8: 557-559.

5. Lang, B., Meske, S., Billmann, P., Vaith, P. \& Peter, H.H. Spontaneous pneumoperitoneum bei progressiver systemsklerose. Z Rheumatol 1986, 45: 64-67.

6. Ritchie, M., Caravelli, J. \& Shike, M. Benign persistent pneumoperitoneum in scleroderma. Dig Dis Sci 1986, 31: $552-555$. 